\title{
Economic Implications of Potential Changes to Regulatory and Reimbursement Policies for Medical Devices
}

\author{
Shelby D. Reed, $P h D^{1,2}$ Alisa M. Shea, $\mathrm{MPH}^{7}$, and Kevin A. Schulman, MD',2,3 \\ ${ }^{1}$ Center for Clinical and Genetic Economics, Duke Clinical Research Institute, PO Box 17969, Durham, NC 27715, USA; ${ }^{2}$ Department \\ of Medicine, Duke University School of Medicine, Durham, NC, USA; ${ }^{3}$ Health Sector Management Program, The Fuqua School of Business, \\ Duke University, Durham, NC, USA.
}

OBJECTIVE: To evaluate the impact of regulatory scenarios on the financial viability of medical device companies.

DESIGN: We developed a model to calculate the expected net present value of a hypothetical product throughout preclinical development, clinical testing, regulatory approval, and postmarketing. We tested 3 scenarios: (1) the current regulatory environment; (2) a scenario in which medical devices are subject to the same evidence standards required for pharmaceuticals; and (3) a scenario consistent with the Coverage with Evidence Development: Coverage with Study Participation (CSP) policy proposed by the Centers for Medicare and Medicaid Services, whereby Medicare will pay for beneficiaries to receive new devices that are not currently determined to be "reasonable and necessary" if the patients participate in clinical studies or registries.

MEASUREMENTS AND MAIN RESULTS: When applying assumptions consistent with the implantable cardioverter-defibrillator market, the net present value at the start of development was an estimated \$553 million in the current regulatory environment, \$322 million in the pharmaceutical scenario, and \$403 million in the CSP scenario. Sensitivity analyses showed that the device industry would likely be profitable in all 3 scenarios over a range of assumptions.

CONCLUSIONS: The environment in which the medical device industry operates is financially attractive. Furthermore, when compared with the alternative of applying the same evidence standards for pharmaceuticals to medical devices, the CSP policy offers improved financial incentives for medical device companies.

KEY WORDS: device approval; health policy; medicare; reimbursement mechanisms.

J Gen Intern Med 23(Suppl 1):50-6

DOI: $10.1007 /$ s1 1606-007-0246-9

(C) Society of General Internal Medicine 2007

\section{INTRODUCTION}

Since the inception of the Medicare program, reimbursement for products and services has been based on whether these are considered "reasonable and necessary for the diagnosis or treatment of illness or injury". ${ }^{1}$ However, as rapid advances in health care technology continue to drive medical costs upward, it has become increasingly difficult for the Centers for Medicare and Medicaid Services (CMS) to balance its dual responsibilities of protecting the Medicare trust fund, while simultaneously enhancing the welfare of beneficiaries-particularly when it comes to coverage decisions for costly new medical devices. ${ }^{2}$

The level of evidence supporting the safety and efficacy of medical devices is typically less than that available for pharmaceutical products. ${ }^{3}$ For high-risk devices or new devices for which there is no comparator product on the market (class III devices), manufacturers must submit a premarket approval application to the Food and Drug Administration (FDA), from which regulators determine whether there is sufficient evidence of safety and effectiveness for the intended uses. ${ }^{4}$ In practice, this standard is often met by small clinical trials in select groups of patients. The studies often do not employ randomized designs, and the FDA generally does not require manufacturers to collect long-term efficacy data. ${ }^{5-7}$ Although there have been improvements in study designs over recent years, and experts have called for increased standardization for medical devices, the FDA Modernization Act of 1997 requires that the FDA allow manufacturers to use the least burdensome means available to demonstrate safety and efficacy. ${ }^{8}$

From the perspective of device manufacturers, increasing the amount of clinical evidence required for approval or reimbursement would create a barrier to market entry. Manufacturers argue that the device industry is fundamentally different from the pharmaceutical industry in terms of organization size and access to capital, and that the engineering framework supporting continuous device innovation stands in contrast to the pharmaceutical industry's focus on the development and testing of drugs. At the same time, the US medical device industry is estimated to be a $\$ 74.5$ billion enterprise, ${ }^{9}$ with substantial firms having dominant positions in critical markets. Thus, the challenge for CMS is to craft a standard for the reimbursement of medical devices that both protects patient safety and preserves the incentive structure that has spurred growth and innovation in the device industry.

To address these and other issues, CMS has proposed the "Coverage with Evidence Development" policy, which includes "Coverage with Appropriateness Determination" (CAD) and "Coverage with Study Participation" (CSP). ${ }^{10}$ In the context of devices, the purpose of CAD is to collect additional data to document that the use of a device accords 
with Medicare's coverage criteria. Under CSP, if CMS determines that the information necessary for a coverage determination is not available, Medicare will reimburse for new devices only if patients enroll in clinical studies or registries supported by the developers of the technologies or other related groups. ${ }^{11-13}$ With these data, Medicare will be better positioned to make evidence-based determinations of whether new devices are "reasonable and necessary". ${ }^{14}$

Although device manufacturers are likely to resist mandates to collect additional data or other requirements that add uncertainty to the reimbursement process, it is also likely that the CSP strategy would be more economically attractive than a policy requiring manufacturers to provide the higher level of evidence usually required of pharmaceutical products in approval and reimbursement decisions. In this paper, we use a simple model to evaluate the financial viability of a hypothetical company developing a new class III medical device in 3 regulatory scenarios that reflect different policy approaches to this issue.

\section{METHODS}

The model calculates the expected net present value (NPV) of a new device throughout preclinical development, clinical testing, regulatory approval, and postmarketing. The NPV of a project is the sum of the present values of all cash flows related to the project, both negative (costs) and positive (revenues). ${ }^{15}$ Firms commonly use NPV calculations to evaluate investment opportunities by explicitly incorporating a stated rate of return that reflects the cost of capital to the firm (known as the discount rate). These discount rates can reflect the availability of capital to the firm, the risk of the investments undertaken by the firm, or even the stage of the investment. In risky endeavors like drug development, in which firms can accrue costs for several years before receiving revenues, future revenues are discounted heavily in the calculation of NPV.

Our model focuses on the NPV of a product at the beginning of the first year of its development (year 1). For simplicity, and to evaluate threshold levels, we dichotomized this measure: If the expected NPV in year 1 is positive, investment in the device is considered economically attractive; if the expected NPV is negative, the company or its investors will be better off directing capital to other investments. (However, if capital or management resources are constrained, the firm would focus on projects with the greatest possible return for a given level of risk.)

\section{Regulatory Scenarios}

The 3 regulatory scenarios of interest are national coverage policies that we have termed the "current scenario", the "CSP scenario", and the "pharmaceutical scenario". The current scenario reflects the current regulatory environment for class III medical devices, in which the level of evidence necessary for FDA approval is less than that required for pharmaceuticals. In this scenario, we assume that CMS will provide payment for a device if it is approved by the FDA, but will not provide payment for the device while it is undergoing clinical testing before approval. In the CSP scenario, CMS will reimburse for the device but will require Medicare beneficiaries who receive the device to be enrolled in a clinical trial or registry. Finally, in the pharmaceutical scenario, CMS will require that the process of developing clinical evidence to justify coverage for a class III medical device is as rigorous as the current FDA approval process for pharmaceutical products.

The structure of our evaluative model is based on the expected life cycle of the device. Compared to pharmaceutical products, it is often difficult to discern when the life cycle of a device begins and ends, because most devices are developed in an incremental fashion. In our model, we assumed that preclinical development of a new device takes approximately 3 years. We assumed that it would take 2 years to complete clinical studies in the current and CSP scenarios and 3 years in the pharmaceutical scenario (Table 1$).{ }^{16}$ For all 3 scenarios, we assumed that CMS is making a national coverage determination, and that it takes the FDA and CMS a total of 1 year to make approval and coverage decisions. ${ }^{17,18}$ Also, because there are fewer regulatory hurdles slowing the entry of other device manufacturers to market, we assumed that the period during which the product can recoup the cost of development is 3 years after clearance from the FDA.

Table 2 summarizes the assumptions of each regulatory scenario. As a starting point, many of the inputs in the model were based on the implantable cardioverter-defibrillator market; however, the model can be applied to numerous medical devices through the use of sensitivity analysis. In the base-case analysis, we assumed that the probability of FDA approval and CMS coverage for the device is $90 \%$ in the current scenario. We applied the same probability to represent the likelihood that CMS would require patients to be enrolled in a registry for the CSP scenario. In the pharmaceutical scenario, we assumed that if the required trial(s) revealed that the device is efficacious, CMS would provide coverage. Otherwise, it would not. We performed extensive sensitivity analyses to evaluate the impact of changes to model parameters and to calculate break-even

Table 1. Device Life Cycle under Three Regulatory Scenarios

\begin{tabular}{|c|c|c|c|c|c|c|c|c|c|c|}
\hline Scenarios & \multicolumn{10}{|c|}{ Year } \\
\hline & 1 & 2 & 3 & 4 & 5 & 6 & 7 & 8 & 9 & 10 \\
\hline Current & \multicolumn{3}{|c|}{ Preclinical development } & \multicolumn{2}{|c|}{ Clinical testing } & $\begin{array}{c}\text { FDA/CMS } \\
\text { review }\end{array}$ & \multicolumn{3}{|c|}{ Sales $(90 \%)$} & - \\
\hline $\mathrm{CSP}$ & \multicolumn{3}{|c|}{ Preclinical development } & Clit & ting & $\begin{array}{c}\mathrm{FDA} / \mathrm{CMS} \\
\text { review }\end{array}$ & \multicolumn{2}{|c|}{ CSP $(90.0 \%)$} & $\begin{array}{c}\text { Sales } \\
(68.5 \%)\end{array}$ & - \\
\hline Pharmaceutical & \multicolumn{3}{|c|}{ Preclinical development } & \multicolumn{3}{|c|}{ Clinical testing } & $\begin{array}{c}\mathrm{FDA} / \mathrm{CMS} \\
\text { review }\end{array}$ & & \multicolumn{2}{|c|}{ Sales $(68.5 \%)$} \\
\hline
\end{tabular}

FDA: Food and Drug Administration; CSP: Coverage with Study Participation. 
Table 2. Assumptions in the Base-case Analysis

\begin{tabular}{|c|c|c|c|}
\hline Assumption & Current Scenario & CSP Scenario & Pharmaceutical Scenario \\
\hline Annual cost of preclinical development ${ }^{a}$ & \$20 million & \$20 million & \$20 million \\
\hline \multicolumn{4}{|l|}{ FDA-mandated studies } \\
\hline Number of patients & $600^{\mathrm{b}}$ & $600^{\mathrm{b}}$ & $2,400^{\mathrm{c}}$ \\
\hline Cost to device company per patient ${ }^{\mathrm{d}}$ & $\$ 25,000$ & $\$ 25,000$ & $\$ 25,000$ \\
\hline \multicolumn{4}{|l|}{ CSP studies } \\
\hline Number of patients ${ }^{\mathrm{e}}$ & - & 20,000 & - \\
\hline Cost to device company per patient $\mathrm{f}^{\mathrm{f}}$ & - & $\$ 1,000$ & - \\
\hline Probability that the device is truly effective ${ }^{g}$ & 0.685 & 0.685 & 0.685 \\
\hline Probability of CMS coverage (traditional or CSP) after FDA approval & $0.900^{\mathrm{h}}$ & $0.900^{\mathrm{h}}$ & $0.685^{\mathrm{g}, \mathrm{i}}$ \\
\hline Probability of CMS coverage after CSP period $^{\mathrm{g}, \mathrm{i}}$ & - & 0.685 & - \\
\hline Patients per year who will receive the device after FDA and CMS approval & 25,000 & 25,000 & 25,000 \\
\hline CMS payment for the device during CSP period ${ }^{\mathrm{k}}$ & - & $\$ 25,000$ & - \\
\hline CMS payment for the device after CMS coverage approval ${ }^{\mathrm{k}}$ & $\$ 25,000$ & $\$ 25,000$ & $\$ 25,000$ \\
\hline Cost to the device company of manufacturing the device ${ }^{1}$ & $\$ 6,000$ & $\$ 6,000$ & $\$ 6,000$ \\
\hline Discount rate $^{\mathrm{m}}$ & $11 \%$ & $11 \%$ & $11 \%$ \\
\hline
\end{tabular}

FDA: Food and Drug Administration; CSP: Coverage with Study Participation, and CMS is Centers for Medicare and Medicaid Services.

${ }^{a}$ Assumption consistent with out-of-pocket costs for preclinical development of pharmaceutical products: \$121 million over 5 years. ${ }^{16}$

${ }^{\mathrm{b}} A$ total of 581 patients were followed for 3 to 6 months for combined cardiac resynchronization therapy and defibrillator (CRT-D; Guidant Corporation).

${ }^{\mathrm{c}}$ Consistent with recommendations from the International Conference on Harmonization, ${ }^{19}$ which suggest that premarket clinical safety databases include 1,500 patients for chronic medications intended to treat non-life-threatening conditions, we assumed that 2 randomized controlled trials with 600 patients per arm are needed for FDA approval in the pharmaceutical scenario.

${ }^{\mathrm{d}}$ Trial-related costs, ${ }^{20}$ not including the cost of manufacturing.

${ }^{\mathrm{e}}$ We assumed that $20 \%$ of patients would choose not to enroll in trials or registries under the CSP policy.

${ }^{\mathrm{f}}$ We assumed that the per-patient cost for CSP incurred by the device company would be significantly lower than costs for clinical trial participation.

${ }^{\mathrm{g}}$ Based on the probability that investigational drugs proceeded from phase 3 testing to marketing approval ${ }^{16}$

${ }^{\mathrm{h}}$ We assumed that $90 \%$ of devices are approved by the FDA.

${ }^{\mathrm{i}}$ We assumed that no type 1 or type 2 errors would occur.

${ }^{\mathrm{j}}$ Estimate based on 64,000 implantable cardioverter defibrillators inserted in 2003, 3 major manufacturers of the devices, and the majority of patients as Medicare beneficiaries ${ }^{21}$

${ }^{\mathrm{k}}$ Assumption consistent with recent estimates of $\$ 18,000$ to $\$ 35,000$ for implantable cardioverter defibrillators ${ }^{22}$

${ }^{1}$ Calculation based on annual report of Guidant Corporation for the cost of all goods as $24 \%(\text { thus, } 0.24 \times \$ 25000=\$ 6000)^{23}$

${ }^{\mathrm{m}}$ Discount rate used by DiMasi et al. ${ }^{16}$

values that correspond to the point at which NPV in year 1 is equal to zero in each scenario.

\section{RESULTS}

In the base case, estimates of NPV in year 1 were $\$ 553$ million in the current scenario, $\$ 403$ million in the CSP scenario, and
$\$ 322$ million in the pharmaceutical scenario, indicating that development of a new device is an economically attractive investment in all 3 scenarios.

In each scenario, device manufacturers spent an estimated $\$ 60$ million on preclinical development over 3 years. They spent an estimated $\$ 18.6$ million over 2 years on clinical testing in the current and CSP scenarios, and \$74.4 million over 3 years in the pharmaceutical scenario. After a 1-year

Table 3. Sensitivity Analyses: Parameter Values When NPV=0

\begin{tabular}{|c|c|c|c|c|}
\hline Variable & Base-case estimate & Current scenario & CSP scenario & Pharmaceutical scenario \\
\hline Annual cost of preclinical development, \$ & 20 million & 223.8 million & 168.7 million & 138.6 million \\
\hline Duration of preclinical development, $\mathrm{y}^{*}$ & 3.00 & 5.47 & 5.32 & 5.04 \\
\hline Probability that device is truly effective, \% & 68.5 & $\mathrm{NA} \dagger$ & $\mathrm{NA} \dagger$ & 16.7 \\
\hline Probability of CMS coverage in current model, \% & 90 & 9.8 & NA & NA \\
\hline Patients receiving device each year after FDA clearance, $n \ddagger$ & 25,000 & 2,709 & 3,038 & 6,083 \\
\hline CMS payment (price) for device, $\$ \S$ & 25,000 & & & \\
\hline 1-year market life & & 7,152 & 10,299 & 15,951 \\
\hline 2-year market life & & 3,743 & 6,011 & 8,142 \\
\hline 3-year market life & & 2,619 & 4,266 & 5,650 \\
\hline 4-year market life & & 2,061 & 3,381 & 4,429 \\
\hline
\end{tabular}

CSP: Coverage with Study Participation; FDA: Food and Drug Administration; CMS: Centers for Medicare and Medicaid Services; and NPV: net present value

*Without varying total life cycle (i.e., with 5 years for clinical development, only 1 year after FDA clearance in each scenario).

$\dagger N P V$ in year 1 is positive at all probabilities. In the current scenario, NPV remains constant under the assumption that evidence is not generated (by trials or through provider experience) to demonstrate ineffectiveness. In the CSP scenario, sufficient sales are generated during the 2 years during CSP to maintain positive NPV regardless of the probability of true effectiveness.

$¥$ In the base-case CSP scenario, 20,000 patients received the device each year during the CSP period, and 25,000 patients received the device each year after FDA clearance. In the break-even analysis, we assumed that the same number of patients received the device in both periods.

$\S$ We maintained the assumption that the cost of goods sold is equal to $24 \%$ of the price of the device. 


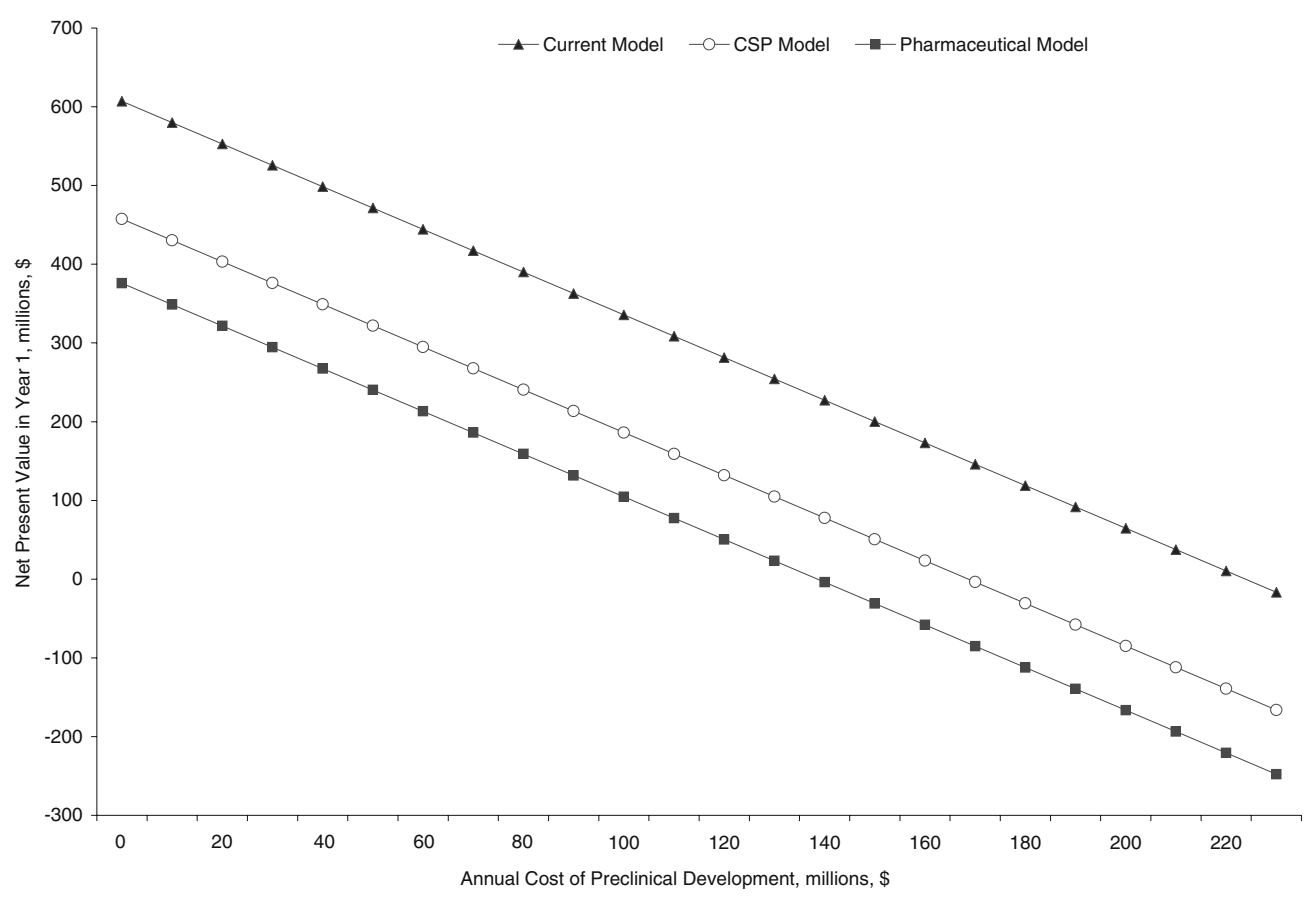

Figure 1. Net present value in year 1 by annual cost of preclinical development

period for FDA and CMS review, expected revenue from CMS for coverage of the device was an estimated \$562.5 million per year for 3 years in the current scenario, with the cost of goods estimated at $\$ 135$ million per year, resulting in net total cash flow of $\$ 1.28$ billion over 3 years. In the CSP scenario, CMS was estimated to pay $\$ 450$ million per year for the 2 years during the CSP period and $\$ 428$ million in the last year of the device's marketable life, with coverage provided by the traditional mechanism. After subtracting the cost of manufacturing the device and the cost of implementing CSP, the net total cash flow was an estimated $\$ 973$ million. Finally, in the pharmaceutical scenario, annual revenues were an estimated $\$ 428$ million per year, with the cost of the devices estimated at $\$ 103$ million per year, resulting in net cash flow of $\$ 976$ million.

\section{Sensitivity Analysis}

Starting with the base-case assumptions for the current scenario, sensitivity analyses showed that payment could be

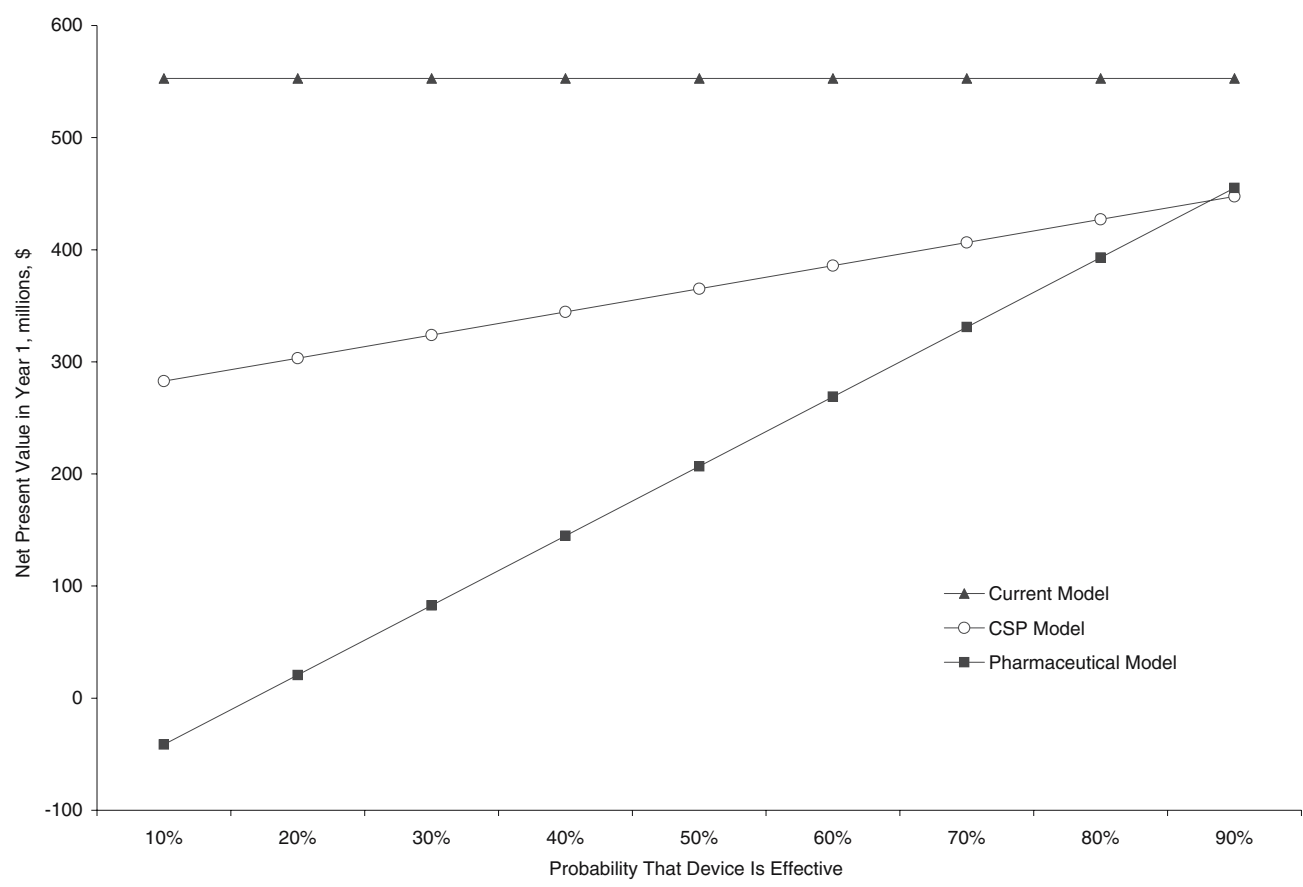

Figure 2. Net present value in year 1 by probability that the device is effective 


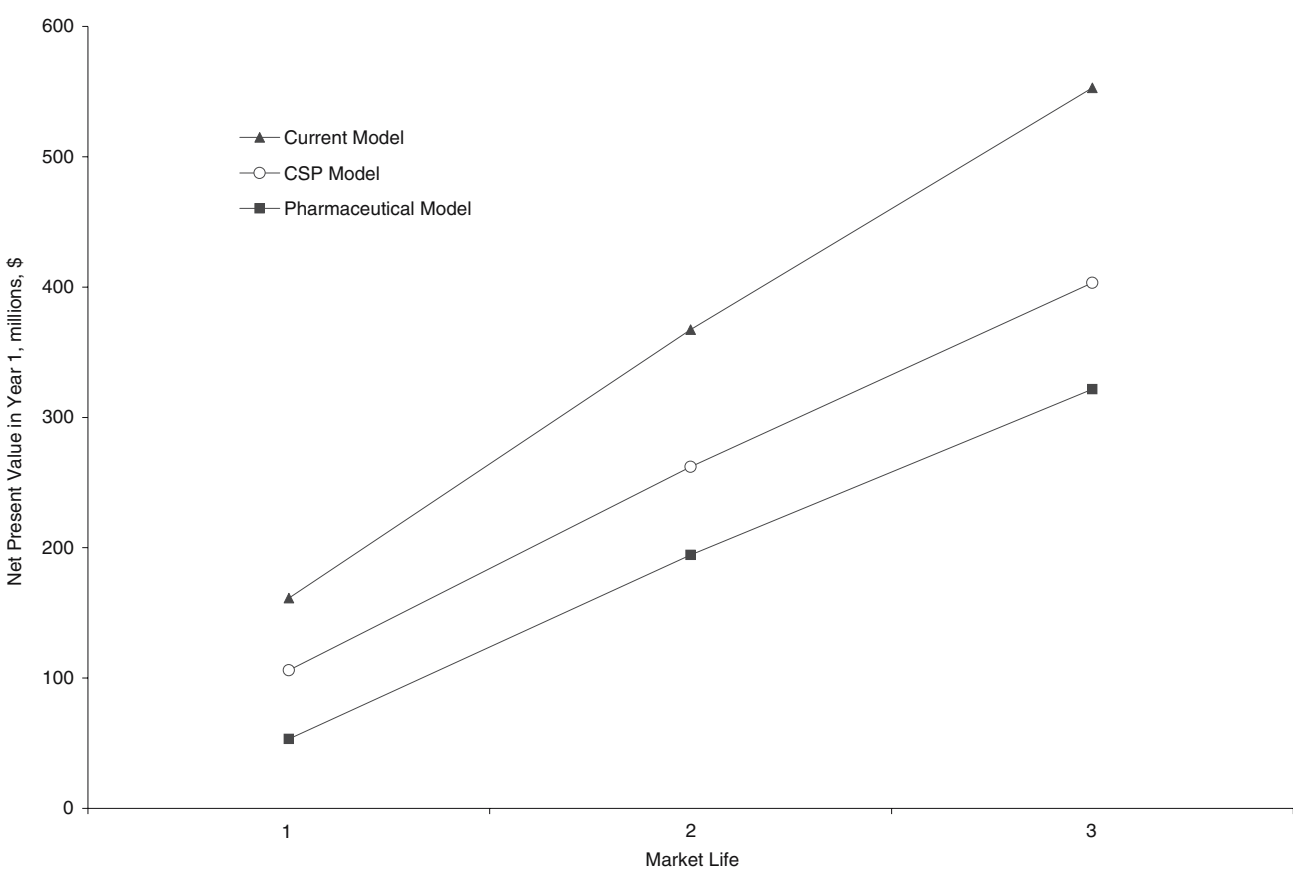

Figure 3. Net present value in year 1 by number of years the device is on market after FDA clearance

as low as about $\$ 2,600$ per device and still allow the NPV to break even at $\$ 0$ (Table 3). Payment could be as low as $\$ 4,266$ per device in the CSP scenario and as low as \$5,650 per device in the pharmaceutical scenario to allow the company to maintain a break-even NPV.

The base-case assumption of $\$ 20$ million in annual costs or $\$ 60$ million in total costs for preclinical development may be an overestimate for devices that represent incremental improvements over existing products, resulting in conservative estimates of NPV; however, this estimate may be too low for new devices. In sensitivity analysis, we found that the annual cost of preclinical development could be as high as \$223.8 million per year in the current scenario, $\$ 168.7$ million per year in the CSP scenario, and $\$ 138.6$ million per year in the pharmaceutical scenario to maintain a positive NPV (Fig. 1 and Table 3).

We also varied the probability that the device would be found truly effective in more extensive clinical testing, as required in the CSP and pharmaceutical scenarios. Given that

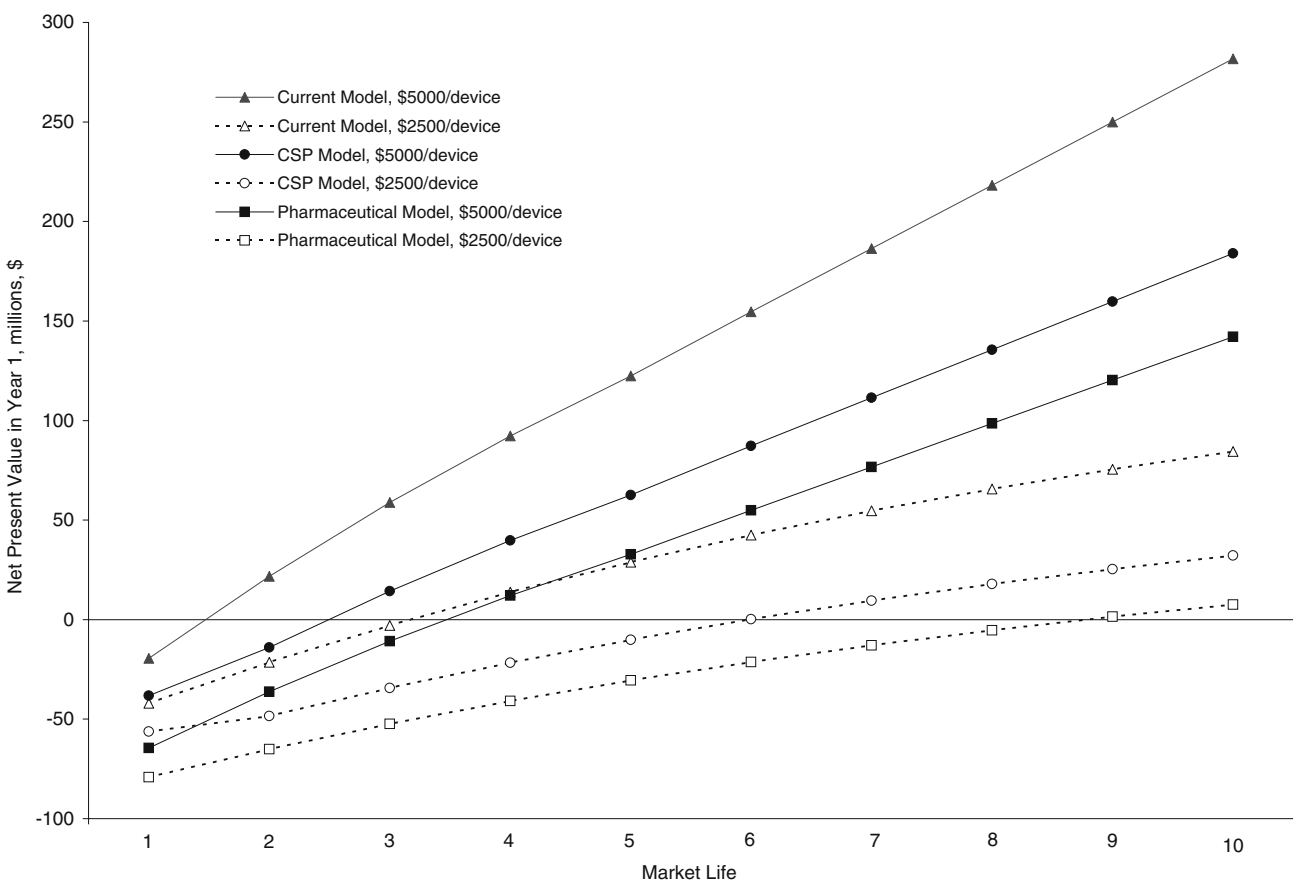

Figure 4. Net present value in year 1 by number of years of active market life at a price of $\$ 5,000$ and $\$ 2,500$ per device 
this probability would only affect the final year of the product's life cycle in the CSP scenario, there was a relatively modest impact on the NPV (Fig. 2). Indeed, the probability that the device was truly effective could be as low as $0 \%$, equivalent to the scenario in which CMS provided coverage for the device only during the CSP period, and the NPV would remain positive. However, when CMS coverage depended entirely on this probability in the pharmaceutical scenario, the NPV decreased by $\$ 62$ million for every $10 \%$ absolute decrease in the probability that the device would be shown effective. The probability could be as low as $16.7 \%$ to maintain a positive expected NPV.

In other sensitivity analyses, we varied the market life of the device. If the device was used in 25,000 patients for 1 or 2 years (rather than 3 years), the NPV in year 1 remained positive (Fig. 3). With a 3-year market life, a manufacturer could maintain a positive NPV in all 3 scenarios with markets as small as approximately 3,000 to 6,000 patients per year (Table 3). Alternatively, if the market life of a product were 10 years, the number of patients receiving the device each year could be as low as approximately 1,400 patients in the CSP scenario and 2,500 patients in the pharmaceutical scenario.

When simultaneously varying the duration of time on the market and the price of the device, we found that if the device was priced at $\$ 5,000$ (instead of $\$ 25,000$ in the base-case analysis), to maintain a positive NPV in year 1 , the product must remain on the market for approximately 1.5 years in the current scenario, 2.5 years in the CSP scenario, and 3.5 years in the pharmaceutical scenario (Fig. 4). If the device was priced at just $\$ 2,500$, the active market life of the device must be at least 3.5 years in the current scenario, 6 years in the CSP scenario, and 9 years in the pharmaceutical scenario (Fig. 4).

Because revenues are a function of price and market size, varying the size of the market was expected to have an impact similar to that of varying the price of the product. If the product was reimbursed at $\$ 25,000$, at least 2,700 patients would have to receive the device annually to maintain a positive NPV in the current scenario, compared to approximately 3,000 patients in the CSP scenario and 6,000 patients in the pharmaceutical scenario.

\section{DISCUSSION}

Our findings suggest that the CSP policy is preferable to a system in which clinical testing for devices is held to the evidence standards currently applied to pharmaceuticals. With CSP, the device industry would benefit from earlier revenue streams generated by the initiation of Medicare coverage for products after relatively limited clinical testing, as compared to delayed revenue streams when products are subject to more rigorous premarket clinical testing.

Sensitivity analyses revealed that the medical device industry is likely to be profitable in all 3 regulatory and reimbursement scenarios over a range of assumptions of lower or higher research and development costs, shorter or longer product cycles, lower or higher prices, and smaller or larger markets. In addition, the assumptions used in our base-case analysis were conservative. For example, we estimated the undiscounted cost of clinical trials to be relatively high in the pharmaceutical scenario, at approximately \$74 million-an amount approaching the $\$ 125$ million estimate for investigational pharmaceuti- cal products. ${ }^{16,18}$ We also assumed that the market life for the device was just 3 years after FDA clearance. And we made the conservative assumption that all FDA-approved devices would be subject to the CSP policy in the CSP scenario. In practice, we expect that the probability will be less than 1, even for class III devices. Although application of CSP is in its early days, it is clear that medical devices will not be exempt. Of the 6 national coverage determinations currently affected by the CED policy, 2 pertain to medical devices (implantable cardioverter-defibrillators and cochlear implants).

The medical device industry is a heterogeneous mix of small new ventures and large mature firms. Larger firms would have the capital necessary to develop a medical device and conduct more rigorous randomized trials to evaluate safety and efficacy, whereas smaller firms may not. Our findings reflect an expected NPV derived from multiplying expected revenues by the probability that firms would receive those revenues. Given that many smaller firms may not be able to sustain a negative approval or coverage decision, the stakes are higher on an individual device basis. That is, although the expected NPV is positive, a lower probability of coverage by CMS will cause a number of small firms to drop out of the marketplace or force them to partner sooner with larger development firms, reducing expected returns to their investors. The ensuing impact on the productivity of research and development across the industry is unknown. It is possible that firms and venture capitalists will become more risk-averse in funding the development of innovative medical devices, or they may selectively inhibit the development of products targeted at smaller patient populations or those with high hurdles to reimbursement.

Although our model is largely hypothetical, it can be used to help shape a discussion of the benefits and tradeoffs to the device industry under various reimbursement models. Nonetheless, some limitations must be considered. First, although we included an estimate of the cost of manufacturing the product, our analysis does not include costs to device companies for sales, marketing, and administration. An accounting of these costs can be considered by reducing reimbursement for the product by an estimate of the percentage of total sales that would be allocated for these activities. For example, in the current scenario, $67 \%$ of sales of the product could be allocated to such expenses while allowing the firm to maintain a positive NPV in year 1 . In the CSP and pharmaceutical scenarios, costs for sales, marketing, and administration could be as high as $63 \%$ and $57 \%$ of sales, respectively, to maintain a positive NPV in year 1. Another limitation is that we did not adjust the cost of capital for the inherent risk of each stage of the research program or additional issues that may affect postapproval uptake of the product. One such issue is whether payments to providers are sufficient to cover the device and associated costs. In some cases, CMS grants additional reimbursements for new technologies through add-on payments for inpatient services, new technology pass-through, or new ambulatory payment classifications for outpatient services. However, this process can take considerable time.

Although we believe our model provides a useful framework for thoughtful discussion about the financial risks imposed on the device market by various regulatory scenarios, the model does not fully address other important issues. One is the value of having high-quality information about risks and benefits of devices after more rigorous clinical testing. Another is the potentially lower risk of adverse events, although this is 
incorporated in our application of a lower probability of a device moving to the sales phase in the CSP and pharmaceutical scenarios. Finally, because the model inherently reflects the perspective of the device industry, it does not incorporate effects on consequent health care spending or the social costs and benefits associated with approving or halting new medical devices.

Overall, our findings substantiate beliefs that, for some markets, the environment in which the medical device industry operates is financially attractive. Furthermore, our analysis shows that when compared with the alternative of applying the same evidence standards for pharmaceuticals to medical devices, the CSP policy offers improved financial incentives for medical device companies.

Acknowledgment: We thank Damon Seils of Duke University for editorial assistance and manuscript preparation.

\section{Funding/Support: None}

Conflict of interest: Drs. Reed and Schulman have received research support from Medtronic, Inc, and Johnson \& Johnson, both manufacturers of medical devices. Dr. Schulman has received personal income from Duke University for teaching in an executive education course sponsored by Stryker Corporation, a manufacturer of medical devices. Ms. Shea reports no conflicts of interest.

Corresponding Author: Kevin A. Schulman, MD; Center for Clinical and Genetic Economics, Duke Clinical Research Institute, PO Box 17969, Durham, NC 27715, USA (e-mail: kevin.schulman@duke. edu).

\section{REFERENCES}

1. Social Security Act, Pub L 74-271, section 1862(a)(1)(A).

2. Foote SB. Why Medicare cannot promulgate a national coverage rule: a case of regula mortis. J Health Polit Policy Law. 2002;27:707-30.

3. Tunis SR, Stryer DB, Clancy CM. Practical clinical trials: increasing the value of clinical research for decision making in clinical and health policy. JAMA. 2003;290:1624-32.

4. Food and Drug Administration. Device Advice: Premarket Approval November 2002. Available at: http://www.fda.gov/cdrh/devadvice/ pma/. Accessed October 23, 2006.

5. Food and Drug Administration. Final Report of the Committee for Clinical Review: Based on a Review of Selected Medical Device Applications. The Temple Report. T93-12. Washington, DC: Food and Drug Administration; March 1993.
6. Kessler L, Ramsey SD, Tunis S, Sullivan SD. Clinical use of medical devices in the "Bermuda Triangle." Health Aff (Millwood). 2004;23:200-7.

7. Maisel WH. Medical device regulation: an introduction for the practicing physician. Ann Intern Med. 2004;140:296-302.

8. Pilot LR, Waldmann DR. Food and Drug Administration Modernization Act of 1997: medical device provisions. Food Drug Law J. 1998;53:267-95.

9. Werner C. Analyst reports medical device market flourishes. Healthc Purch News. 2003;27:30-1.

10. Centers for Medicare and Medicaid Services. National coverage determinations with data collection as a condition of coverage: coverage with evidence development; July 12, 2006. Available at: https://www.cms. hhs.gov/mcd/ncpc_view_document.asp?id=8. Accessed October 23 , 2006.

11. Kolata G. Medicare Covering New Treatments, but with a Catch. New York Times, 5 November 2004.

12. Centers for Medicare and Medicaid Services. Proposed Decision Memo For Positron Emission Tomography (FDG) for Brain, Cervical, Ovarian, Pancreatic, Small Cell Lung, and Testicular Cancers (CAG-00181N), 1 November 2004. Available at: http://www.cms.hhs.gov/mcd/viewdraft decisionmemo.asp?id=92. Accessed October 23, 2006.

13. Centers for Medicare and Medicaid Services. Proposed Decision Memo For Anticancer Chemotherapy For Colorectal Cancer (CAG-00179N), 1 November 2004. Available at: http://www.cms.hhs.gov/mcd/viewdraft decisionmemo.asp?id=90. Accessed October 23, 2006.

14. Centers for Medicare and Medicaid Services. Procedures for Making Coverage Decisions. 64 Federal Register 22619-22625 (27 April 1999).

15. Stickney CP, Weil RL. Financial Accounting: An Introduction to Concepts, Methods, and Uses, 10th ed. Cincinnati, Ohio: South-Western College Publishing; 2003.

16. DiMasi JA, Hansen RW, Grabowski HG. The price of innovation: new estimates of drug development costs. J Health Econ. 2003;22:151-85.

17. Center for Drug Evaluation and Research, Food and Drug Administration. FDA's Drug Review and Approval Times. Available at: http://www.fda gov/cder/reports/reviewtimes/default.htm. Accessed October 23, 2006.

18. Centers for Medicare and Medicaid Services. Review of National Coverage Determinations and Local Coverage Determinations; Final Rule. 68 Federal Register 63692-63731 (7 November 2003).

19. International Conference on Harmonization of Technical Requirements for Registration of Pharmaceuticals for Human Use (ICH). The extent of population exposure to assess clinical safety. ICH-E1A. Available at: http://www.fda.gov/cder/guidance/ichela.pdf. Accessed October 23, 2006.

20. Eisenstein EL, Lemons PW 2nd, Tardiff BE, Schulman KA, Jolly MK, Califf RM. Reducing the costs of phase III cardiovascular clinical trials. Am Heart J. 2005; 149:482-8.

21. Heart disease and stroke statistics-2006 update: a report from the American Heart Association Statistics Committee and Stroke Statistics Subcommittee. Circulation. 2006;113:e85-151. Erratum in: Circulation. 2005; 113:e696.

22. Hlatky MA, Mark DB. The high cost of implantable defibrillators. Eur Heart J. 2006; (doi: 10.1093/eurheartj/ehl311).

23. Guidant Annual Report 2003. Indianapolis: Guidant Corporation; 2003. Available at: http://www.guidant.com/investors/annualreport/2003/ Guidant_AR2003.pdf. Accessed November 18, 2005. 